\title{
DFT studies of CNT-functionalized uracil-acetate hybrids
}

\author{
Mahmoud Mirzaei ${ }^{\mathrm{a}, *}$, Oguz Gulseren ${ }^{\mathrm{b}}$ \\ a Department of Medicinal Chemistry, School of Pharmacy and Pharmaceutical Sciences, Isfahan University of Medical Sciences, Isfahan, Iran \\ ${ }^{\mathrm{b}}$ Department of Physics, Faculty of Science, Bilkent University, Ankara, Turkey
}

\section{H I G H L I G H T S}

- Hybridizations of uracil acetate and CNTs are investigated.

- Electronic and structural properties of molecular functionalized CNTs are investigated.

- Different properties regarding methylated or charged uracil acetate are observed.

\section{A R T I C L E I N F O}

\section{Article history:}

Received 7 March 2015

Received in revised form

13 May 2015

Accepted 19 May 2015

Available online 21 May 2015

Keywords:

Carbon nanotube

Uracil

Density functional theory

Nuclear magnetic resonance

\section{G R A P H I C A L A B S T R A C T}

The molecular functionalizations of a representative CNT by different forms of uracil-acetates have been investigated through DFT calculations.

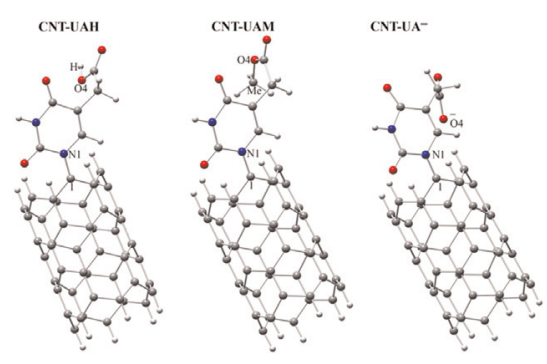

\begin{abstract}
A B S T R A C T
Calculations based on density functional theory (DFT) have been performed to investigate the stabilities and properties of hybrid structures consisting of a molecular carbon nanotube (CNT) and uracil acetate (UA) counterparts. The investigated models have been relaxed to minimum energy structures and then various physical properties and nuclear magnetic resonance (NMR) properties have been evaluated. The results indicated the effects of functionalized CNT on the properties of hybrids through comparing the results of hybrids and individual structures. The oxygen atoms of uracil counterparts have been seen as the detection points of properties for the CNT-UA hybrids.
\end{abstract}

(c) 2015 Elsevier B.V. All rights reserved.

\section{Introduction}

Considerable efforts following the pioneering discovery of carbon nanotubes (CNTs) have yielded that the biological applications could be expected for this novel material [1,2]. Moreover, other nano-based structures with typical characters have been introduced through the attempts of so many researchers in the following years $[3,4]$. Besides the known importance of individual nano-based structures, their hybridizations with other structures

\footnotetext{
*Corresponding author. Fax: +983136680011.

E-mail address: mdmirzaei@pharm.mui.ac.ir (M. Mirzaei).
}

have been viewed important for various types of applications $[5,6]$. Combining the nano-based structures with medicinal species have been expected to be helpful for the process of targeted drug delivery in living systems $[7,8]$. Because of undesired side effects of classical drugs, design and synthesis of novel drugs in combinations with nano-based structures have been proposed as a possible way to reduce their side effects $[9,10]$. The carrier roles of nanobased structures have been also expected to exactly deliver the drugs to the targeted receptor $[11,12]$. Therefore, studies of formations and properties of hybrids of nano-based structures and medicinal species have become the topics of several computational and experimental research works in recent years [13-16]. In 


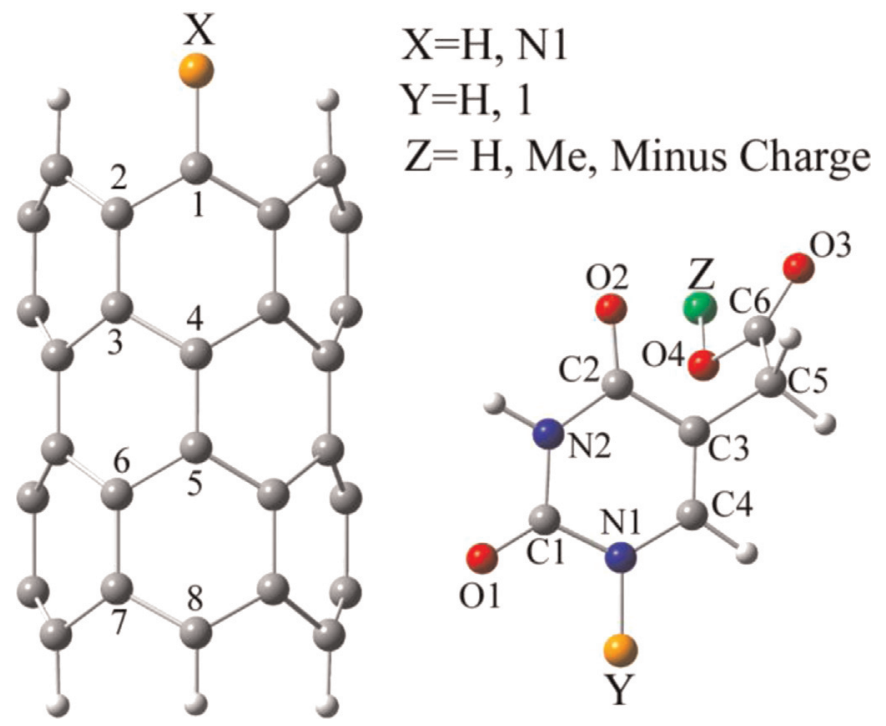

Fig. 1. The counterparts (CNT in left and UA in right) of hybrid compounds are shown.

addition to the benefits of nano-based structures for medicinal purposes, the hydrophobicity of non-polar nano-materials e.g., CNTs, could be significantly reduced in contribution with biological hybrids [17]. Since the hydrophobic nano-materials could not be easily dispersed in solutions, a biological coverage could help them to overcome the problem [18]. Although experimental measurements are essential, computational methods could reveal insightful information on the atomic and molecular levels of investigated subjects prior to complicated experiments $[19,20]$.

Within this work, formations and properties of CNT-functionalized uracil-acetate compounds (Figs. 1 and 2) have been investigated through the quantum chemical computations. The carbon atom number five (C5) of uracil, the characteristic nucleobase of ribonucleic acid (RNA), is a proper atomic site to be functionalized by other atomic and molecular groups for medicinal applications [21]. Flurouracil, in which the C5 position is functionalized by a fluorine atom, is a highlighted uracil derivative applicable in cancer therapies for a long time up to now [22]. The C5 position is functionalized by an acetate group to make another biologically important uracil derivative, the uracil-acetate (UA) compound. Kalhor and Clarke [23] showed that the UA structures are important for their unique actions in the processes of methyltransferases in eukaryotic tRNA of biological systems. Moreover, they achieved different properties for hydrogenated (UAH), methylated (UAM), and negatively charged $\left(\mathrm{UA}^{-}\right)$related compounds. In this work, the properties for all three available compounds have been investigated to show the effects of existence of a CNT counterpart on their original characteristics (Fig. 1). To make the CNT-UA hybrid, a representative CNT molecular group is attached to the nitrogen atom number one (N1) of UA structure, which is initially the atomic site of ribose sugar group attachment in the uridine nucleotide [24]. The constructed CNT-UA hybrids including CNT-UAH, CNT-UAM, and CNT-UA ${ }^{-}$compounds (Fig. 2) have been computationally investigated through geometry optimizations and properties evaluations to compare with parallel characteristics in the individual structures (Tables 1 and 2). The major question of this work is to show the effects of CNT functionalizations on the original properties of UA derivatives, which is tried to be investigated by the computationally evaluated results.

\section{Computational details}

In this study, the CNT-functionalized uracil-acetate hybrids are investigated density functional theory (DFT). All the calculations have been performed using the B3LYP exchange-correlation functional and the $6-31 \mathrm{G}^{*}$ standard basis set as implemented in the Gaussian 98 program [25]. First, all model structures of this work including CNT, UAH, UAM, and $\mathrm{UA}^{-}$individual compounds and CNT-UAH, CNT-UAM, and CNT-UA ${ }^{-}$hybrids (Figs. 1 and 2) have been fully optimized to reach their minimum energies. As described previously [5], thevalues of total energies, binding energies, energy gaps, and dipole moments (Table 1) have been evaluated for the model structures with this optimization process. Since the model structures are chemically bonded together through covalent bonds, the interferer existence of basis set superposition error (BSSE), which is important for physical
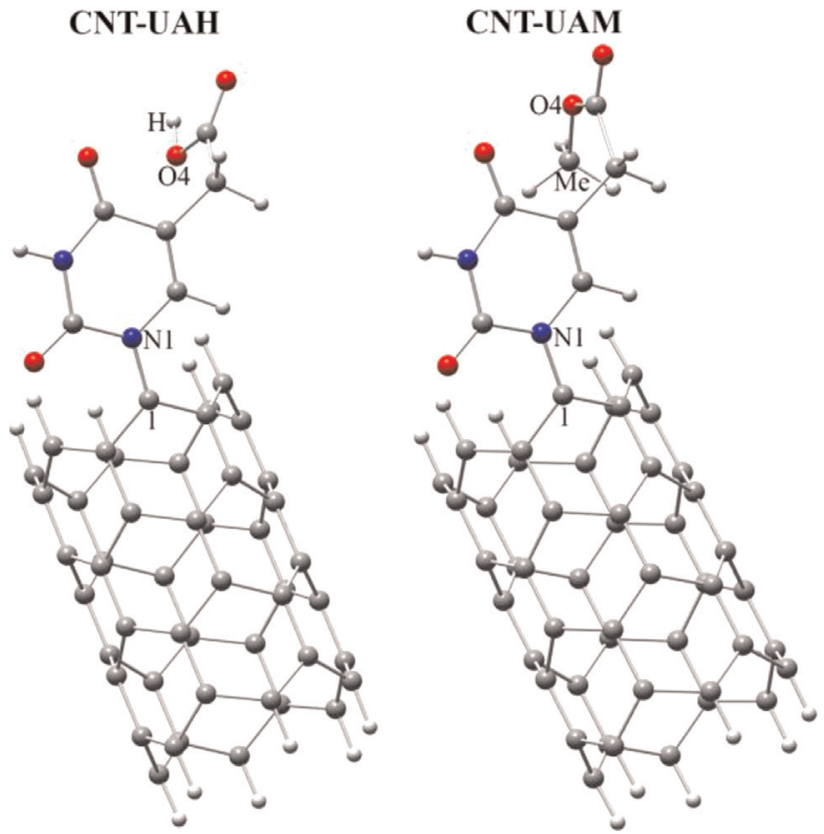

\section{CNT-UA}

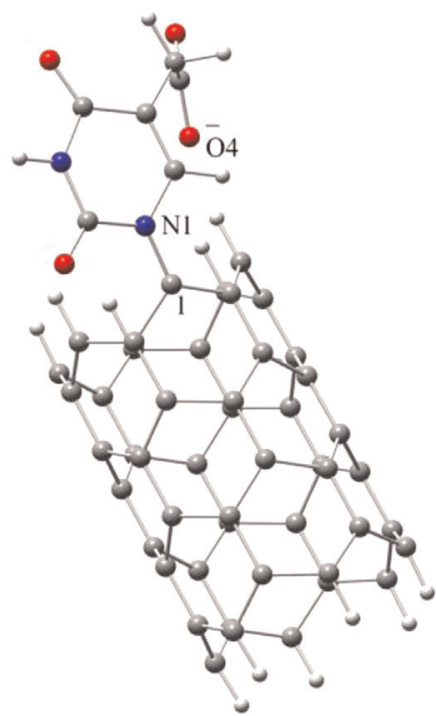

Fig. 2. The investigated CNT-UA hybrids are shown. 
Table 1

Various physical properties from optimized structures of CNT-UA hybrids*.

\begin{tabular}{|c|c|c|c|c|}
\hline Property & {$[\mathrm{CNT}]$} & $\begin{array}{l}\text { CNT-UAH } \\
\text { [UAH] }\end{array}$ & $\begin{array}{l}\text { CNT-UAM } \\
\text { [UAM] }\end{array}$ & $\mathrm{CNT}^{-\mathrm{UA}^{-}}\left[\mathrm{UA}^{-}\right]$ \\
\hline Stoichiometry & {$\left[\mathrm{C}_{48} \mathrm{H}_{12}\right]$} & $\begin{array}{l}\mathrm{C}_{54} \mathrm{H}_{16} \mathrm{~N}_{2} \mathrm{O}_{4} \\
{\left[\mathrm{C}_{6} \mathrm{H}_{6} \mathrm{~N}_{2} \mathrm{O}_{4}\right]}\end{array}$ & $\begin{array}{l}\mathrm{C}_{55} \mathrm{H}_{18} \mathrm{~N}_{2} \mathrm{O}_{4} \\
{\left[\mathrm{C}_{7} \mathrm{H}_{8} \mathrm{~N}_{2} \mathrm{O}_{4}\right]}\end{array}$ & $\begin{array}{l}\mathrm{C}_{54} \mathrm{H}_{15} \mathrm{~N}_{2} \mathrm{O}_{4} \\
{\left[\mathrm{C}_{6} \mathrm{H}_{5} \mathrm{~N}_{2} \mathrm{O}_{4}\right]}\end{array}$ \\
\hline \multirow{2}{*}{$\begin{array}{l}\text { Total energy } \\
\quad / \mathrm{keV}\end{array}$} & {$[-49.958]$} & -67.415 & -68.484 & -67.400 \\
\hline & & {$[-17.489]$} & {$[-18.558]$} & {$[-17.474]$} \\
\hline \multirow{2}{*}{$\begin{array}{l}\text { Binding energy/ } \\
\mathrm{eV}\end{array}$} & {$[-361.283]$} & -442.977 & -454.861 & -442.012 \\
\hline & & {$[-87.033]$} & {$[-99.285]$} & {$[-85.835]$} \\
\hline $\mathrm{HOMO} / \mathrm{eV}$ & {$[-3.567]$} & $\begin{array}{l}-3.892 \\
{[-7.023]}\end{array}$ & $\begin{array}{l}-3.863 \\
{[-6.884]}\end{array}$ & $\begin{array}{l}-1.434 \\
{[-1.026]}\end{array}$ \\
\hline LUMO/eV & {$[-3.125]$} & $\begin{array}{l}-3.462 \\
{[-1.763]}\end{array}$ & $\begin{array}{l}-3.432 \\
{[-1.495]}\end{array}$ & $\begin{array}{l}-1.001 \\
{[-2.578]}\end{array}$ \\
\hline Energy gap/eV & [0.442] & $\begin{array}{l}0.430 \\
{[5.260]}\end{array}$ & $\begin{array}{l}0.431 \\
{[5.389]}\end{array}$ & $\begin{array}{l}0.433 \\
{[1.552]}\end{array}$ \\
\hline \multirow{2}{*}{$\begin{array}{l}\text { Dipole moment/ } \\
\text { Debye }\end{array}$} & [0.003] & 9.444 & 10.168 & 13.791 \\
\hline & & [6.258] & [6.966] & [9.278] \\
\hline
\end{tabular}

* See Figs. 1 and 2 for the models. The values in brackets and also for CNT are in the singular structures. The other values are for hybrid structures.

Table 2

Quadrupole coupling constants $\left(C_{Q} / \mathrm{kHz}\right)$; Chemical shifts $(\delta / \mathrm{ppm})^{*}$.

\begin{tabular}{|c|c|c|c|c|}
\hline Atom & {$[\mathrm{CNT}]$} & CNT-UAH [UAH] & CNT-UAM [UAM] & $\mathbf{C N T}^{-\mathbf{U A}^{-}}\left[\mathbf{U A}^{-}\right]$ \\
\hline 1 & {$[1366 ; 127]$} & $1950 ; 107$ & $1815 ; 110$ & $1709 ; 120$ \\
\hline 2 & {$[1761 ; 151]$} & $1272 ; 139$ & $1243 ; 138$ & $1131 ; 142$ \\
\hline 3 & {$[1407 ; 137]$} & $1560 ; 145$ & $1542 ; 145$ & $1371 ; 137$ \\
\hline 4 & {$[1533 ; 142]$} & $1189 ; 137$ & $1227 ; 137$ & $1292 ; 140$ \\
\hline 5 & {$[1532 ; 142]$} & $1676 ; 144$ & $1647 ; 144$ & $1559 ; 141$ \\
\hline 6 & {$[1407 ; 137]$} & $1215 ; 131$ & $1246 ; 132$ & $1408 ; 140$ \\
\hline 7 & {$[1761 ; 151]$} & $1734 ; 150$ & $1754 ; 151$ & $1898 ; 153$ \\
\hline 8 & {$[1363 ; 127]$} & $1851 ; 131$ & $1672 ; 128$ & $645 ; 124$ \\
\hline $\mathrm{C} 1$ & - & $\begin{array}{l}1492 ; 136 \\
{[1723 ; 138]}\end{array}$ & $\begin{array}{l}1546 ; 137 \\
{[1730 ; 138]}\end{array}$ & $\begin{array}{l}1592 ; 140 \\
{[1827 ; 140]}\end{array}$ \\
\hline $\mathrm{C} 2$ & - & $\begin{array}{l}2322 ; 152 \\
{[2402 ; 151]}\end{array}$ & $\begin{array}{l}2309 ; 148 \\
{[2383 ; 151]}\end{array}$ & $\begin{array}{l}2648 ; 150 \\
{[2729 ; 154]}\end{array}$ \\
\hline $\mathrm{C} 3$ & - & $\begin{array}{l}699 ; 103 \\
{[698 ; 104]}\end{array}$ & $\begin{array}{l}802 ; 105 \\
{[609 ; 106]}\end{array}$ & $\begin{array}{l}1435 ; 115 \\
{[1162 ; 114]}\end{array}$ \\
\hline $\mathrm{C} 4$ & - & $\begin{array}{l}2320 ; 129 \\
{[2285 ; 132]}\end{array}$ & $\begin{array}{l}2287 ; 129 \\
{[2176 ; 130]}\end{array}$ & $\begin{array}{l}2392 ; 127 \\
{[3265 ; 137]}\end{array}$ \\
\hline $\mathrm{C} 5$ & - & $\begin{array}{l}752 ; 39 \\
{[807 ; 36]}\end{array}$ & $\begin{array}{l}815 ; 37 \\
{[813 ; 37]}\end{array}$ & $\begin{array}{l}920 ; 41 \\
{[1040 ; 31]}\end{array}$ \\
\hline C6 & - & $\begin{array}{l}2630 ; 156 \\
{[2635 ; 154]}\end{array}$ & $\begin{array}{l}2617 ; 155 \\
{[2608 ; 155]}\end{array}$ & $\begin{array}{l}2484 ; 150 \\
{[2563 ; 156]}\end{array}$ \\
\hline N1 & - & $\begin{array}{l}2916 ; 170 \\
{[3862 ; 127]}\end{array}$ & $\begin{array}{l}3062 ; 167 \\
{[3912 ; 126]}\end{array}$ & $\begin{array}{l}3029 ; 172 \\
{[3784 ; 125]}\end{array}$ \\
\hline N2 & - & $\begin{array}{l}3520 ; 155 \\
{[3637 ; 155]}\end{array}$ & $\begin{array}{l}3580 ; 156 \\
{[3643 ; 155]}\end{array}$ & $\begin{array}{l}3626 ; 155 \\
{[3660 ; 156]}\end{array}$ \\
\hline 01 & - & $\begin{array}{l}8556 ; 310 \\
{[8378 ; 297]}\end{array}$ & $\begin{array}{l}8555 ; 310 \\
{[8323 ; 291]}\end{array}$ & $\begin{array}{l}8421 ; 306 \\
{[7964 ; 256]}\end{array}$ \\
\hline $\mathrm{O} 2$ & - & $\begin{array}{l}8289 ; 308 \\
{[9399 ; 242]}\end{array}$ & $\begin{array}{l}9289 ; 246 \\
{[9348 ; 242]}\end{array}$ & $\begin{array}{l}9429 ; 249 \\
{[9132 ; 277]}\end{array}$ \\
\hline $\mathrm{O} 3$ & - & $\begin{array}{l}9614 ; 239 \\
{[9339 ; 245]}\end{array}$ & $\begin{array}{l}9696 ; 210 \\
{[9712 ; 209]}\end{array}$ & $\begin{array}{l}8169 ; 304 \\
{[8151 ; 314]}\end{array}$ \\
\hline $\mathrm{O} 4$ & - & $\begin{array}{l}8098 ; 194 \\
{[8564 ; 179]}\end{array}$ & $\begin{array}{l}10225 ; 161 \\
{[10221 ; 161]}\end{array}$ & $\begin{array}{l}6766 ; 252 \\
{[7765 ; 300]}\end{array}$ \\
\hline
\end{tabular}

* See Figs. 1 and 2 for the models. The values in brackets are for the singular structures. The other values are for the hybrid structures. In each column, the first number is for the $C_{Q}$ parameters and the second one is for the $\delta$ parameters $\left(C_{Q} ; \delta\right)$. interacting systems, is neglected. The values of total energies have been directly obtained by DFT calculations as the overall nuclear and electronic energies. To obtain the values of binding energies, the differences between the magnitudes of total energies and atomic energies have been considered e.g., Binding Energy $(\mathrm{CNT}-\mathrm{UAH})=E_{\mathrm{CNT}-\mathrm{UAH}}-54 E_{\mathrm{C}}-16 E_{\mathrm{H}}-2 E_{\mathrm{N}}-4 E_{\mathrm{O}}$. The differences between the calculated magnitudes of energies for the highest occupied molecular orbital (HOMO) and the lowest unoccupied molecular orbital (LUMO) have been considered as the values for molecular energy gaps. To better recognize the properties of investigated structures at the atomic levels, nuclear magnetic resonance (NMR) properties including quadrupole coupling constants $\left(C_{Q}\right)$ and chemical shifts $(\delta)$ (Table 2) have been subsequently evaluated for the atoms of optimized structures as described elsewhere [26]. Sincethe NMR properties are originated from the electronic sites of atoms, they could reveal insightful information about the characteristics of matters [27].

\section{Results and discussion}

\subsection{The optimized properties}

The evaluated optimized properties including total energies, binding energies, energy gaps, and dipole moments for the investigated model structures of CNT, UAH, UAM, UA' ${ }^{-}$, CNT-UAH, CNT-UAM, and CNT-UA' (Figs. 1 and 2), which are defined in the Introduction section, are summarized in Table 1 . The hydrogen atom of nitrogen atom number one (N1) of UA counterpart and that of carbon atom number one (C1) of CNT counterpart have been removed to make chemical functionalization processes for the investigated hybrids. Earlier studies indicated that both chemical and physical functionalizations could be observed for the hybrids of CNTs and biological counterparts [28,29]. The employed representative CNT is a $(6,0)$ zigzag nanotube with the stoichiometry of $\mathrm{C}_{48} \mathrm{H}_{12}$, in which the hydrogen atoms are used to saturate the atoms of tubular tips to avoid dangling effects [30].

Examining the values of Table 1 reveals reasonable changes of magnitudes for total energies among the models due to different number of atoms for molecular structure. The magnitudes of binding energies, which are released by chemical bonds in structural formations, reveal more favorable formations of CNT-UAM and UAM models than other hybrids and individuals. The magnitudes of energy gaps, referring to the energy differences between the HOMO and LUMO levels reveal similar orbital properties for three hybrids whereas those of UA models are different. It could be mentioned that the existence of CNT could modulate the orbital energy levels of the CNT-UA hybrids whereas different orbital energy levels are observed for the individual UA models. However, a deeper look at the values of HOMO and LUMO levels show that the exact magnitudes of energies for the frontier molecular orbitals are different among the CNT-UA hybrids but the distances between the HOMO and LUMO levels are similar. It could be referred to the modulating property of the CNT for the conducting properties of CNT-UA hybrids. The magnitudes of dipole moments reveal that the polarities are significantly arisen in the hybrids in comparison with the individual models. The polarity of CNT-UA ${ }^{-}$ is the largest one among the hybrids whereas those of other two hybrids are almost close to each other. As a conclusion for this section, it could be mentioned that the molecular properties are different for the individual and hybrid models. The CNT could modulate the orbital energy levels, but the binding energies and dipole moments indicate that the CNT-UA ${ }^{-}$is less stable than the two other hybrids. The binding energies and dipole moments reveal parallel results for $\mathrm{UA}^{-}$in comparison with UAH and UAM. And finally, the CNT could influence on the molecular properties of 
UA models as shown by the evaluated optimized properties.

\subsection{The NMR properties}

The values of quadrupole coupling constants $\left(C_{Q}\right)$ and chemical shifts $(\delta)$ for the atoms of optimized individual and hybrid model structures (Figs. 1 and 2) are listed in Table 2. Both mentioned properties are obtained by the nuclear magnetic resonance (NMR) spectroscopy, which is among the most versatile characterization techniques of matters [27]. Besides the complicated experimental measurements, quantum chemical computations could also yield reliable values of $C_{Q}$ and $\delta$ properties, in which their evaluations are described in an earlier work [26]. The interaction energies between the $z$-axis eigenvalue $\left(q_{z z}\right)$ of electric field gradient (EFG) tensors and the nuclear electric quadrupole moment $(Q)$ are measured by $C_{Q}$ properties [27]. The arisen EFG tensors at the electronic sites of atoms could show any perturbations employed to these sites as measured by the $C_{Q}$ properties. The discrepancies of average magnitudes of eigenvalues of isotropic chemical shieldings $\left(\sigma_{i i}\right)$ for the sample form the reference model are measured by $\delta$ properties to show the amount of changes of electron density at the atomic sites [27]. The $C_{Q}$ value implies for a direct measurement of electronic magnitudes at the atomic sites whereas the $\delta$ implies for changes of average electron densities with respect to a reference value. The structures of $\mathrm{Si}\left(\mathrm{CH}_{3}\right)_{4}, \mathrm{H}_{2} \mathrm{O}$, and $\mathrm{NH}_{3}$ are respectively used for the $\delta$ references of carbon, oxygen, and nitrogen atoms. Computationally obtaining the atomic and molecular properties could be helpful for better engineering the molecular systems to achieve desired purposes at the low levels, which is especially followed in nano-related technologies.

A quick examination of the obtained values of $C_{Q}$ and $\delta$ properties (Table 2) indicates that the effects of chemical environments are remarkably characterized in the investigated model structures. The properties for the individual CNT model show similarities at two tubular tips whereas the similarity is interrupted in the hybrid models due to effects of attached UA counterpart. Interestingly, the properties of CNT are also different for the three hybrid systems due to different types of attached UAH, UAM, and $\mathrm{UA}^{-}$. A deeper examination shows that the changes of properties for CNT-UA ${ }^{-}$ hybrid are more significant than two other UAH and UAM hybrids. The CNT-UA ${ }^{-}$model is negatively charged; therefore, the minus charge remarkably changes the properties for this model in comparison with the other models. Comparing the properties for atoms of single UA structures indicates that the most significant changes are observed for the atoms of acetate regions, in which the type of acetate group differs by the attached hydrogen atom, methyl group, or a minus charge. The properties for the carbon atoms of different positions in the UA counterparts are still different in comparison with each other. For the nitrogen atoms, N1 plays the important role of linking the UA counterparts to the CNT group due to its initial character of attachment to ribose sugar in the uridine nucleotide [24]. Comparing the properties for N1 and N2 atoms shows only slight changes for N2 atom during the hybridizations whereas the changes for $\mathrm{N} 1$ atom are many more significant in the same way. In the original single UA structures, the N1 is hydrogenated; however, the hydrogen atom is replaced by the carbon atom number one (C1) of CNT through a covalent $\mathrm{N}-$ $\mathrm{C}$ bond. Therefore, the changes of properties for N1(UA) and C1 (CNT) atoms are significant among other atoms. There are two sets of oxygen atoms in the UA structures, in which $\mathrm{O} 1$ and $\mathrm{O} 2$ belong to the pyrimidine ring whereas $\mathrm{O} 3$ and 04 belong to the acetate group. Different properties are observed for the mentioned atoms in the single and hybrid models. The properties of $\mathrm{O} 1$ atoms are different in the single UA structures; however, it seems that their properties are modulated in the CNT-UA hybrids with a slight difference only for CNT-UA ${ }^{-}$hybrid. The changes of properties of
$\mathrm{O} 2$ atoms are more significant than $\mathrm{O} 1$ atoms among the single and hybrid models. For the acetate group, the $\mathrm{O} 3$ atom is the ketotype oxygen, which keeps its own position among the UA models. Examining the properties for $\mathrm{O} 3$ atoms reveals only slight changes for the electronic properties of this atom during the hybridization process. It could be proposed that the properties of $\mathrm{O} 3$ could be remained almost unchanged in the hybrid models in comparison with the single model. The $\mathrm{O} 4$ atom is the driving atom of UA models, which divides them into three forms of UAH, UAM, and $\mathrm{UA}^{-}$models. With the exception of UAM model, the changes of properties from the single model to hybrids are significant for $\mathrm{O} 4$ atoms. Although this atomic site is far from the attached CNT region, its properties still detect the effects of hybridizations as could be seen by the evaluated properties. It is worth noting that the magnitudes of changes for the $C_{Q}$ and $\delta$ properties for each atom in different structural states could reveal the magnitude of changes for its electronic properties in the states. Moreover, the values of $C_{Q}$ could indicate the electronic properties of atoms in each individual structure, in which insightful trends could be obtained especially for examining the capabilities of atomic sites for interactions with other atoms or molecules for determining their further applications.

\section{Conclusion}

The results of our DFT calculations indicated that the stabilities for CNT-UAM and UAM formations are more favorable than other models. Moreover, the influences of CNT existence on the properties of hybrids have been seen through changes of HOMO-LUMO energy gaps and dipole moments. For better clarifications, the evaluated NMR properties indicated that the $\mathrm{N}_{1}$ atom significantly detects the effects of changes of hybridizations whereas the effects for $\mathrm{N}_{2}$ are almost negligible. However, the changes for oxygen atoms are more significant than other atoms; therefore, the properties of oxygen atoms could be the detection points of the investigated CNT-UA hybrids.

\section{References}

[1] S. Iijima, Helical microtubules of graphitic carbon, Nature 354 (1991) 56-58.

[2] R.V. Mundra, X. Wu, J. Sauer, J.S. Dordick, R.S. Kane, Nanotubes in biological applications, Curr. Opin. Biotechnol. 28 (2014) 25-32.

[3] D. Cakır, O. Gulseren, First-principles study of thin $\mathrm{TiO}_{x}$ and bulklike rutile nanowires, Phys. Rev. B 80 (2009) 125424.

[4] M.B. Javan, M.D. Ganji, Theoretical investigation on the encapsulation of atomic hydrogen into heterofullerene nanocages, Curr. Appl. Phys. 13 (2013) 1525-1531.

[5] M. Mirzaei, H.R. Kalhor, N.L. Hadipour, Investigating purine-functionalised carbon nanotubes and their properties: a computational approach, IET Nanobiotechnol. 5 (2011) 32-35.

[6] M.S. Ali, H.A. Al-Lohedan, M.Z.A. Rafiquee, A.M. Atta, A.O. Ezzat, Spectroscopic studies on the interaction between novel polyvinylthiol-functionalized silver nanoparticles with lysozyme, Spectrochim. Acta Part A: Mol. Biomol. Spectrosc. 135 (2015) 147-152.

[7] S. Mohammadi-Samani, R. Miri, M. Salmanpour, N. Khalighian, S. Sotoudeh, N. Erfani, Preparation and assessment of chitosan-coated superparamagnetic $\mathrm{Fe}_{3} \mathrm{O}_{4}$ nanoparticles for controlled delivery of methotrexate, Res. Pharm. Sci. 8 (2013) 25-33.

[8] F. Farvadi, A.M. Tamaddon, S.S. Abolmaali, Z. Sobhani, G.H. Yousefi, Micellar stabilized single-walled carbon nanotubes for a pH-sensitive delivery of doxorubicin, Res. Pharm. Sci. 9 (2014) 1-10.

[9] S. Liu, Epigenetics advancing personalized nanomedicine in cancer therapy, Adv. Drug Deliv. Rev. 64 (2012) 1532-1543.

[10] J.P. Jee, J.H. Na, S. Lee, S.H. Kim, K. Choi, Y. Yeo, I.C. Kwon, Cancer targeting strategies in nanomedicine: design and application of chitosan nanoparticles, Curr. Opin. Solid State Mater. Sci. 16 (2012) 333-342.

[11] M. Amoli-Diva, K. Pourghazi, M.H. Mashhadizadeh, Magnetic pH-responsive poly(methacrylic acid-co-acrylic acid)-co-polyvinylpyrrolidone magnetic nano-carrier for controlled delivery of fluvastatin, Mater. Sci. Eng.: C 47 (2015) 281-289.

[12] H. Chaudhary, K. Kohli, V. Kumar, A novel nano-carrier transdermal gel against 
inflammation, Int. J. Pharm. 465 (2014) 175-186.

[13] M. Kharaziha, S.R. Shin, M. Nikkhah, S.N. Topkaya, N. Masoumi, N. Annabi, M. R. Dokmeci, A. Khademhosseini, Tough and flexible CNT-polymeric hybrid scaffolds for engineering cardiac constructs, Biomaterials 35 (2014) $7346-7354$.

[14] C. Rieger, D. Kunhardt, Anika Kaufmann, Darja Schendel, Doreen Huebner, Kati Erdmann, Stefan Propping, Manfred P. Wirth, Bernd Schwenzer, Susanne Fuessel, Silke Hampe, Characterization of different carbon nanotubes for the development of a mucoadhesive drug delivery system for intravesica treatment of bladder cancer, Int. J. Pharm. 479 (2015) 357-363.

[15] M. Mirzaei, Effects of carbon nanotubes on properties of the fluorouracil anticancer drug: DFT studies of a CNT-fluorouracil compound, Int. J. Nano Dimens. 3 (2013) 175-179.

[16] S.Y. Kim, J.Y. Hwang, J.W. Seo, U.S. Shin, Production of CNT-taxol-embedded PCL microspheres using an ammonium-based room temperature ionic liquid: as a sustained drug delivery system, J. Colloid Interface Sci. 442 (2015) 147-153.

[17] E.V. Gorbachev, M.G. Kiselev, N.A. Fomina, Effect of the polarity of functiona groups $(-\mathrm{H},-\mathrm{OH}$, and $-\mathrm{COOH})$ on the structure of a solvent near single-wal carbon nanopipes, Russ. J. Phys, Chem. A 85 (2011) 2192-2196.

[18] S. Park, H.S. Yang, D. Kim, K. Job, S. Jon, Rational design of amphiphilic polymers to make carbon nanotubes water-dispersible, anti-biofouling, and functionalizable, Chem. Commun. 25 (2008) 2876-2878.

[19] E. Zurek, J. Autschbach, Density functional calculations of the 13C NMR Chemical Shifts in (9,0) single-walled carbon nanotubes, J. Am. Chem. Soc. 126 (2004) 13079-13088.

[20] G. Wu, S. Dong, R. Ida, N. Reen, A solid-state 170 nuclear magnetic resonance study of nucleic acid bases, J. Am. Chem. Soc. 124 (2002) 1768-1777.

[21] K. Kramer, P. Hummel, H.H. Hsiao, X. Luo, M. Wahl, H. Urlaub, Mass-spectrometric analysis of proteins cross-linked to 4-thio-uracil- and 5-bromo-uracilsubstituted RNA, Int. J. Mass Spectrom. 304 (2011) 184-194.

[22] M. Nasr, M.K. Ghorab, A. Abdelazem, In vitro and in vivo evaluation of cubosomes containing 5-fluorouracil for liver targeting, Acta Pharm. Sin. B 5 (2015)
79-88.

[23] H.R. Kalhor, S. Clarke, Novel methyltransferase for modified uridine residues at the wobble position of tRNA, Mol. Cell. Biol. 23 (2003) 9283-9292.

[24] T. Partovi, M. Mirzaei, N.L. Hadipour, The C $-\mathrm{H}$. . . O hydrogen bonding effects on the 170 electric field gradient and chemical shielding tensors in crystalline 1-methyluracil: a DFT study, Z. Naturforsch. A 61 (2006) 383-388.

[25] M.J. Frisch, G.W. Trucks, H.B. Schlegel, G.E. Scuseria, M.A. Robb, J.R. Cheeseman, V.G. Zakrzewski, J.A. Montgomery, R.E. Stratmann, J.C. Burant, S. Dapprich, J.M. Millam, A.D. Daniels, K.N. Kudin, M.C. Strain, O. Farkas, J. Tomasi, V. Barone, M. Cossi, R. Cammi, B. Mennucci, C. Pomelli, C. Adamo, S. Clifford, J. Ochterski, G. A. Petersson, P.Y. Ayala, Q. Cui, K. Morokuma, D.K. Malick, A.D. Rabuck, K. Raghavachari, J.B. Foresman, J. Cioslowski, J.V. Ortiz, A.G. Baboul, B.B. Stefanov, G. Liu, A. Liashenko, P. Piskorz, I. Komaromi, R. Gomperts, R.L. Martin, D.J. Fox T. Keith, M.A. Al-Laham, C.Y. Peng, A. Nanayakkara, C. Gonzalez, M. Challacombe, P.M.W. Gill, B. Johnson, W. Chen, M.W. Wong, J.L. Andres, C. Gonzalez, M. Head-Gordon, E.S. Replogle, J.A. Pople, Gaussian 98, Revision A.7, Gaussian, Inc, Pittsburgh, PA, 1998

[26] M. Mirzaei, N.L. Hadipour, Study of hydrogen bonds in 1-methyluracil by DFT calculations of oxygen, nitrogen, and hydrogen quadrupole coupling constants and isotropic chemical shifts, Chem. Phys. Lett. 438 (2007) 304-307.

[27] R.S. Drago, Physical Methods for Chemists, second ed., Saunders College Publishing, 1992.

[28] R. Rafiee, R. Pourazizi, Influence of CNT functionalization on the interphase region between CNT and polymer, Comput. Mater. Sci. 96 (2015) 573-578.

[29] M. Mirzaei, M. Meskinfam, M. Yousefi, A cytosine-assisted carbon nanotubes junction: DFT studies, Superlattices Microstruct. 52 (2012) 158-164.

[30] Z. Bagheri, M. Mirzaei, N.L. Hadipour, M.R. Abolhassani, Density functional theory study of boron nitride nanotubes: calculations of the N-14 and B-11 nuclear quadrupole resonance parameters, J. Comput. Theor. Nanosci. 5 (2008) 614-618. 